\title{
A Modular Speed-Drooped System for High Reliability Integrated Modular Motor Drives
}

\author{
Alessandro Galassini, \\ Alessandro Costabeber, Chris Gerada \\ PEMC Group, The University of Nottingham \\ United Kingdom, China \\ alessandro.galassini@nottingham.ac.uk
}

\author{
Giampaolo Buticchi \\ Chair of Power Electronics \\ University of Kiel \\ Germany \\ gibu@tf.uni-kiel.de
}

\author{
Davide Barater \\ University of Parma \\ Italy \\ davide.barater@unipr.it
}

\begin{abstract}
Future transportation challenges include a considerable reduction in pollutant emissions at a time when significant increase in demand is predicted. One of the enabling solutions is the electrification of transport systems as this should lead to improved operability, fuel savings, emission reduction and maintenance. Whilst state-of-the-art technology has demonstrable benefits there needs to be considerable advancement to meet future transportation affordability and emission targets. Primarily, electrical drives need an improved power density, an increased reliability and a reduced specific cost. For this reason, Integrated Modular Motor Drives (IMMD) present an attractive solution. Modularity leads to redundancy and easier integration. This paper presents a novel speed-drooped control system applied to motors fed by modular paralleled converters. This control technique allows precise speed regulation and power sharing among different segments showing improved fault tolerance and reliability. The design procedure and the power sharing dynamic have been presented and analysed by means of Matlab/Simulink and validated in a $3 \mathrm{~kW}$ experimental rig, showing good agreement with the expected performances.
\end{abstract}

Index Terms-Drives, electrical machines, speed-droop, parallel converters, voltage source converter (VSC).

\section{INTRODUCTION}

Nowadays most three-phase motors are supplied by a conventional three phase-motor drive [1]. Next generation drives should provide greater reliability, robustness and competitiveness on the global market of propulsion systems. A segmented machine is one of the possible solutions to increase fault tolerance, giving the chance to redesign the drive concept, providing the power in a modular and integrated way. Integrated Modular Motor Drives (IMMD) are the result of this process of innovation suitable for applications requiring high efficiency and power density [2], [3]. Redesign of the drive with the power electronics building block (PEBB) [4] in mind is not just a simple physical redistribution of the components into the housing to save space and wire length, but it is a chance to investigate new possible features, and therefore, new market opportunities. At the moment, higher cost is the main issue preventing their diffusion into the market, but the flexibility and functionalities introduced by IMMD systems is expected to reduce the payback time in the near future; indeed, due to their modularity, on a large scale economy the costs of the IMMD will be cut down [1].
Modularisation introduces redundancy, increasing up time service and availability. This new kind of approach leads to new functionalities, for example, depending on the winding and configuration, the drive could detect a fault in a segment and reconfigure the system to cope with the fault condition [5]-[8]. On the other hand, integration adds some problems, for example, thermal management; consequently more emphasis must be taken in heat extraction [9], [10]. Currently only a few prototypes of modular and/or integrated drives have been developed [3], [11]-[15]. Due to their benefits listed in Table I, IMMDs are one of the best candidates for future electrical propulsion systems [16].

TABLE I

PROS AND CONS OF IMMD

\begin{tabular}{|c|c|}
\hline Benefits & Drawbacks \\
\hline Volume, Weight $\Downarrow$ & Design complexity in general \\
Wires length, Costs $\Downarrow$ & Thermal management \\
Electro Magnetic Interference $\Downarrow$ & Control strategy \\
Redundancy and Reliability $\Uparrow$ & Mechanical integration \\
Availability and Maintainability $\Uparrow$ & Vibrations suppression \\
\hline
\end{tabular}

Among the possible choices, this paper considers the specific case of a rewound three phase machine, where two main arrangements are possible: series and parallel, shown in Fig. $1 \mathrm{a}$ and Fig. 1b respectively [2], [3]. In both the figures, the DC/AC blocks are meant to be the three phase two level inverter topology (Fig. 1c). Each inverter is connected to the relative three phase set of windings of a multi-three phase electrical motor. The arrangement obviously affects the system behaviour and, consequently, the control system. The main limitation of the series configuration (Fig. 1a) is the fault tolerance; in fact, if a segment is damaged, the entire system is compromised. On the other hand, in the parallel configuration (Fig. 1b), due to the replication of the paralleled inverters and the three-phase sets of windings, both in case of segment or drive fault, system service is guaranteed. For this reason between the two configurations, the parallel one is the most suitable for IMMD, therefore it has been considered here. The other relevant design choice is the connection of the neutral points. If they were connected together, in case of a fault, the 


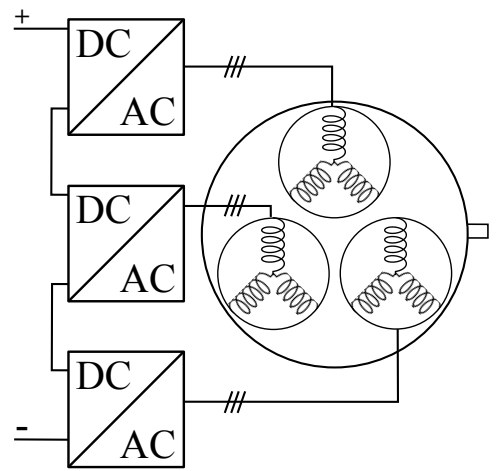

(a) Series inverters

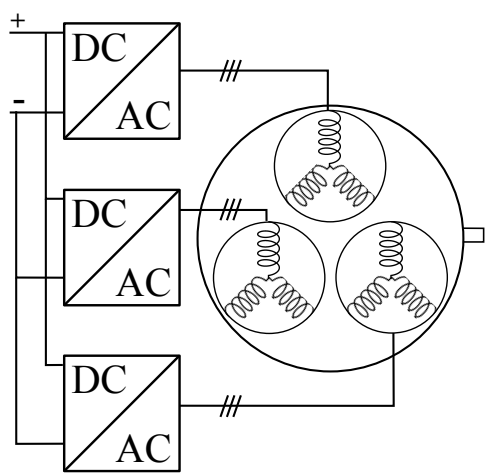

(b) Paralleled inverters

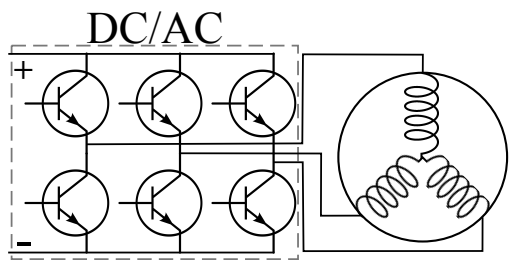

(c) Inverter - set of windings connection

Fig. 1. Different multi-drive arrangements for the two level three-phase inverter

system could be reconfigured thanks to additional switches and modified modulation strategies [5], [17]. Even if the three phase systems are isolated, fault tolerant strategies have been developed and verified [6]. Since the connection of the neutral points increases the complexity and establishes the flow of undesired circulating currents between segments, the neutral points will be considered disconnected.

In literature different strategies for multi-drive systems have been proposed (Fig. 2), and their main features are summarised in Sec. II. As an alternative, this paper proposes an enhanced droop based controller for the IMMD which guarantees tightly regulated speed while arbitrarily sharing the load power among the modules [18], [19]. In the proposed technique, the load sharing ratio can be dynamically adjusted with predictable response. The details of the proposed control solution are presented in Sec. III. Sec. IV outlines a simplified procedure for control design and Sec. V discusses the impact of droop slopes on the current sharing dynamics. The overall design approach is summarised in Sec. VI, whereas a case study is presented in Sec. VII. The validation of the proposed analysis including simulations and experimental results is provided in Sec. VIII.

\section{Multi Drive Control Strategies}

Nowadays, power sharing in drive applications (like for example conveyor systems, large diameter bull gears with multiple drives, printing presses with in-line drive shaft, coal cars, cement kilns, and separator drums) are achieved thanks to mainly three different control techniques: common speed reference (Fig. 2a), torque follower (Fig. 2b) and speed trim follower (Fig. 2c) [20], [21]. The common speed reference is the simplest where all the drives are operated in speed mode. The power sharing ratio cannot be changed, thus power is always equally split among the segments. Since all the drives are independent, the main advantage of such a configuration is its fault tolerance. In the torque follower configuration (Fig. 2b) interconnections between drives are required [22], [23], and for this reason, it is not modular and not fault tolerant. However, both the load sharing and the speed control are precise. The master drive is operated in speed regulation,

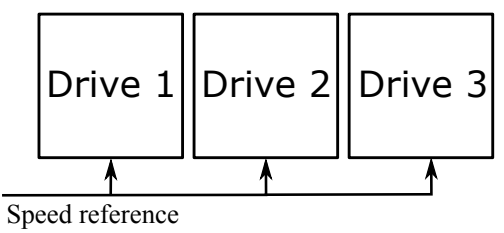

(a) Common speed reference

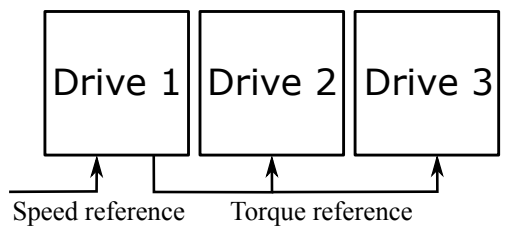

(b) Torque follower

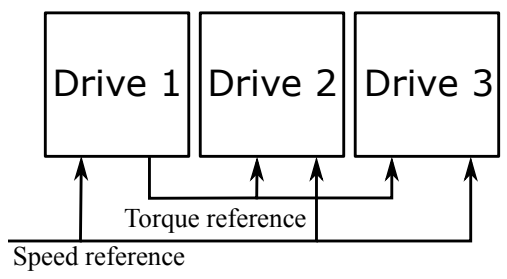

(c) Speed trim follower

Fig. 2. Different multi-drive control strategies

whereas the followers are operated in torque regulation mode. This layout is used where coupling among the motors is rigid and speed regulation is critical. In the speed trim follower all the drives are operated with speed regulation (Fig. 2c). Whilst the master takes speed reference as it is provided, the followers are added with a trimmed speed. The trim adjusts the speed set-point comparing the local torque set-point with the one from the master. This guarantees equal torque generated by each module. With rigid couplings the torque set-points are the same and no adjustments are encountered. The speed trim follower configuration is adopted when the coupling among motors has a very high potential for oscillation. Since each drive is fed with the torque reference of the master drive, the speed trim follower is not modular. Even if its layout could be reconfigured in case of fault thanks to a supervisory controller, 


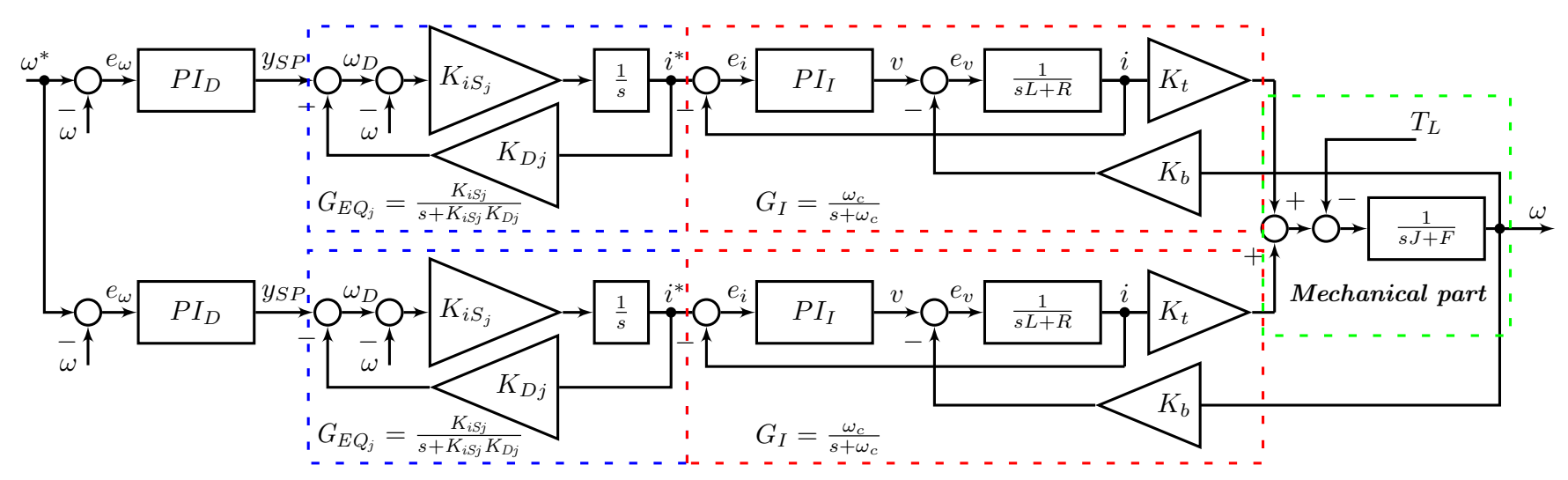

Fig. 3. Drooped control schematic with two modules, thus the motor should be intended as a double three-phase machine.

complexity would increase compromising reliability.

Among the control strategies mentioned above, the only one having independent drives is the the common speed reference. For this reason, it is more suitable for IMMD compared to the torque follower and the speed trim follower configuration. However, the common speed reference does not allow a precise load sharing [20], [21]. The proposed speed droop controller allows a precise load sharing even if all the drives are configured in speed mode and being independent one from each other at the same time.

The aforementioned configuration can be used to control paralleled converters connected to a single machine with one rotor and multiple three phase stator windings, or when using more than one independent machines with the rotors directly coupled together with stiff joints. Since actuation power is shared among different modules with independent power electronics and isolated windings, if the system is properly reconfigured under fault conditions, the overall reliability is improved. Since various faults may occur for very different systems, they will not be listed here. In general, as soon as the fault is detected thanks to the current fault detection techniques for three-phase AC motor drives [24], the system will be able to operate at reduced power, unless the individual modules are overrated to accommodate one or more failures in the system. In the next section the developed speed droop controller is introduced and detailed.

\section{THE PROPOSED SPEED DROOP CONTROLLER}

From a general perspective, droop control is a very well known technique used in power systems to share power demanded by the grid among different generation systems [25], [26], in uninterruptible power supply (UPS) [27], and DC/DC converter [28] scenarios. In AC power system scenario, the basic droop characteristic is a linear function with a negative coefficient on the Frequency-Active power plane governed by the following equation [29]:

$$
\omega_{i}=\omega_{0}-K_{D} P_{i}
$$

where $\omega_{i}$ and $\omega_{0}$ are the angular frequency of the output voltage and of the base reference respectively, $P_{i}$ is the output

active power, and $K_{D}$ is the droop coefficient. The greater the

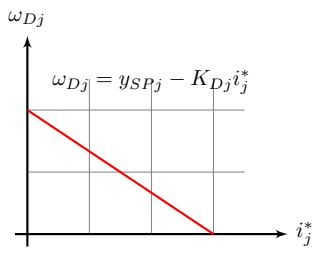

(a) Droop plane

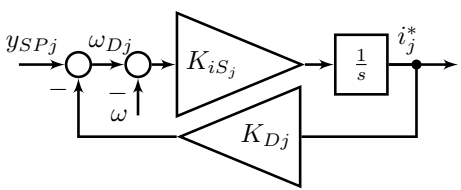

(b) Droop controller $\left(G_{E Q j}\right)$ implementation with speed feedback

Fig. 4. Droop characteristic

frequency the less is the power produced by the generation plant. Changing the coefficients of individual power stations sets the power produced by each one of them. Thanks to this, the way power injected into the grid is partitioned among generation plants is a function of the droop coefficients [30].

Translating these concepts into an IMMD like the one in Fig. $1 \mathrm{~b}$ power sharing among the drives is possible. The key point here is the droop plane (Fig. 4a). Generation systems are defined by angular frequency $\omega$ and the active power $P$, however IMMD are described by the current set-point $i^{*}$ of a single $j$-inverter and the internal speed reference $y_{S P j}$ like the following (Fig. 4b):

$$
\omega_{D j}=y_{S P j}-K_{D j} i_{j}^{*}
$$

Indicating the generic inverter (or module) of the IMMD with $j$, Fig. 3 shows how the droop has been implemented in the proposed speed droop system. Even if the same approach can be applied to an IMMD with a generic number $n$ of modules, in Fig. 3 the full control scheme is reported for $n=2$. The mechanical load is common and represented with a linear model with inertia $J$ and friction $F$. The two threephase windings are represented by a generalised scheme of the q-axis of the machine with stator resistor $R$ and the stator inductor along the q axis $L$. This is to highlight that the control approach can be applied to any machine, replacing $K_{b}$ and $K_{t}$ with the specific back emf and torque equations. The regulator $P I_{I}$ is the current controller. The outer regulator, $P I_{D}$, is the 
compensation PI with the goal of restoring the desired speed set-point and to fulfil the speed dynamic constraints (Fig. 5).

In order to better understand the droop controller $G_{E Q_{j}}$ (Fig. 4b) between the current PI and the compensation PI, consider first a simple integral speed controller $\left(K_{D j}=0\right)$ where the regulator $K_{i S_{j}} / s$ processes the speed error with respect to a constant reference $\omega_{D j}=y_{S P j}$, and defines the current set-point $i_{j}^{*}$. Introducing the droop coefficients $K_{D j}$, the speed set-point $\omega_{D j}$ is now a function of the ideal setpoint $y_{S P j}$ minus a component dependent on the actual current (torque) demand. The correlation among values is reported in Fig. $4 \mathrm{a}$ and the droop regulator in Fig. $4 \mathrm{~b}$ can be written as:

$$
G_{E Q j}(s)=\frac{K_{i S j}}{s+K_{i S j} K_{D j}}=\frac{i_{j}^{*}(s)}{y_{S P j}(s)}
$$

If we assume an ideal inner current loop, i.e. $i_{j}=i_{j}^{*}$, and

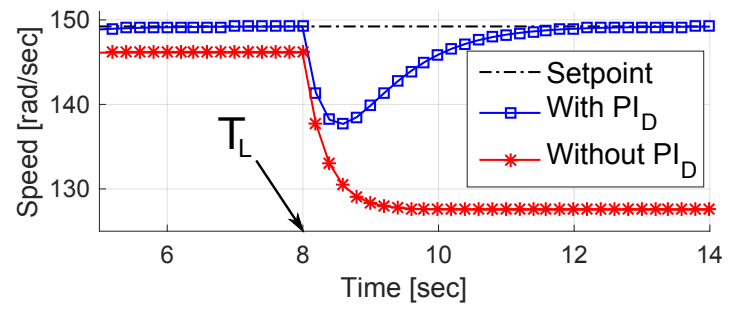

Fig. 5. With compensation PI the final speed tracks the set-point. At second $8^{t h}$ the load $\left(T_{L}=17 \mathrm{Nm}\right)$ is attached and the speed error increases like described by (4).

we assume constant speed set-point $y_{S P j}=\omega^{*}$, the steady state speed error resulting from the inner drooped loops can be calculated from Fig. 3 taking the limit for $s \rightarrow 0$ :

$$
\begin{array}{r}
\omega=\omega^{*} \frac{\gamma}{\gamma+1}-\frac{T_{L}}{F+\varepsilon K_{t}} \\
\text { with } \varepsilon=\sum_{j}^{n} \frac{1}{K_{D j}} \text { and } \gamma=\frac{\varepsilon K_{t}}{F}
\end{array}
$$

As expected, the droop causes an error on the speed $\omega$, and the compensation PI $\left(P I_{D}\right)$ has been introduced to restore the speed set-point $\omega^{*}$ (Fig. 5), by shifting the droop characteristics up or down to guarantee that the steady state equilibrium in Eq. (4) matches the set-point $\omega^{*}$.

In a previous work, other researchers [31] developed a similar drooped-speed control for mitigating the 0 -sequence current between two paralleled drives connected to a three phase machine, but without compensating the error introduced by the droop coefficient. In the proposed procedure the droop loop has been taken into account from the beginning, compensating the final speed and guaranteeing desired performances.

\section{SimplifiEd EQUiVALENT MODEL}

Assuming at frequencies of interest the integrator of droop regulator $I_{S j}$ has a gain which is high enough to assume

$$
\left|I_{S j}\right|=\left|\frac{K_{i S j}}{s}\right|>>K_{D_{j}}
$$

an intuitive understanding of the system operation can be better gained. Under the condition in (5) the scheme in Fig. 3 can be simplified into a system where the compensation PI $P I_{D}$ remains in place but all the inner loops with droop, including $K_{i S j}, K_{D j}$ and the inner adder, can be replaced by a gain $1 / K_{D j}=W_{j}$ receiving the output of $P I_{D}$, and directly providing the current reference for the inner current controller $P I_{I}$ (Fig. 6). All the control parameters and the machine parameters are assumed to be the same in each module, and only the droop coefficients can vary to change the power sharing. This simplified approach where the droop is reduced to a set of parallel PI controllers $\left(P I_{D}\right)$ with different gains $\left(1 / K_{D j}=W_{j}\right)$ depending on the desired sharing ratio would be the simplest approach for a system operating with constant sharing ratio. Such a kind of arrangement would be

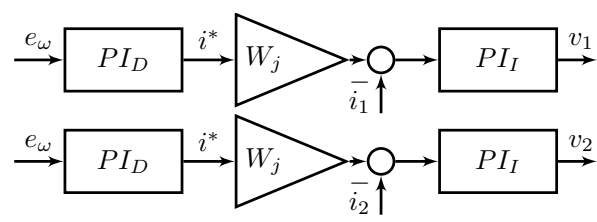

Fig. 6. Simplified droop under condition in (5)

like a common speed reference system with a tunable current set-point. However in this case, a change in the coefficients $W_{j}$ would be instantaneously reflected in a change of the references for the current loops, causing a poorly damped sharing transient leading to torque stresses and undesired vibrations. In the control structure proposed in this paper, one of the desired features is to be able to control the current sharing dynamic after sharing ratio step changes. In this case, the droop implementation using the scheme in Fig. 4b instead of a simple gain like in Fig. 6 guarantees smoother transients after step changes in power sharing demand.

Assuming that the load power is equally split among the $n$ modules, the schematic in Fig. 3 can be simplified with the collective one in Fig. 7, where the parallel current controllers have been replaced by the closed loop function with bandwidth $\omega_{c}$. The parallel of the $n$ droop controllers $G_{E Q j}^{(E S)}$ (where $(E S)$

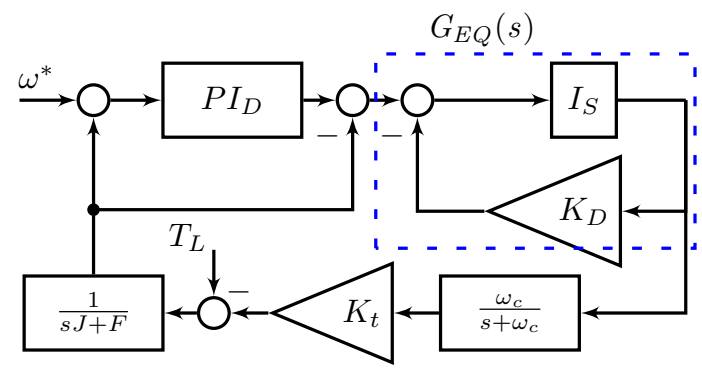

Fig. 7. Equivalent collective control scheme valid when the load power is equally split among the $n$ modules. $I_{s}=K_{i S} / s$.

stands for equal sharing) has been replaced by an equivalent collective controller:

$$
G_{E Q}=n G_{E Q j}^{(E S)}
$$


The equality in Eq. (6) can be satisfied if and only if $K_{i S}=$ $K_{i S j}^{(E S)} n$ and $K_{D}=K_{D j}^{(E S)} / n$ as highlighted by Eq. (7):

$$
G_{E Q}(s)=\frac{n K_{i S j}^{(E S)}}{s+K_{i S j}^{(E S)} n \frac{K_{D j}^{(E S)}}{n}}=n G_{E Q j}^{(E S)}(s)
$$

From the above equation it can be observed that the whole system can be modelled as an equivalent single module with integral gain $K_{i S}$ and droop gain $K_{D}$ :

$$
K_{i S}=K_{i S_{j}}^{(E S)} n \quad K_{D}=\frac{K_{D_{j}}^{(E S)}}{n}
$$

The $K_{D}$ and the $K_{i S}$ gain in Eq. (8) can be defined as the collective droop and the collective integral gain coefficient respectively.

\section{DROOP SLOPES AND CURRENT SHARING DYNAMIC}

The equivalent collective model (Fig. 7) built on the previous section has assumed power equally shared among the $n$ modules of the IMMD. The total power $P_{T O T}$ is given by the sum of all the nominal torques produced by each module multiplied by the shaft speed. Since currents and torques are directly proportional $\left(T=K_{t} i\right)$, the $j$-th power in p.u. is described by the following:

$$
P_{j}=\frac{I_{n o m, j}}{\sum_{j}^{n} I_{n o m, j}}[p . u .]
$$

where $I_{n o m, j}$ is the nominal current on the q-axis of the $j$-th module. From Fig. 3, it can be noticed that the current setpoints are the output of the sharing regulators $G_{E Q_{j}}$. Provided that in the steady state the magnitude of the droop loops is the reciprocal of the droop coefficient $\left|G_{E Q_{j}}\right|_{s \rightarrow 0}=1 / K_{D}$, the total power $P_{T O T}$ can be described as $\varepsilon$ (the sum of the reciprocals as previously defined). Therefore, the power provided by each of the $n$ modules in general (not only when the power is equally split) can be written as:

$$
P_{j}=\frac{1 / K_{D j}}{\sum_{j}^{n}\left(1 / K_{D j}\right)}=\frac{1 / K_{D j}}{\varepsilon}[p . u .]
$$

Thanks to Eq. (10), as long as $\varepsilon$ is kept constant, unbalanced power sharing can be achieved just changing the droop coefficients. Whenever a different sharing ratio is needed (not equal sharing like previously supposed), the droop coefficients $K_{D j}$ could be updated by a supervisory controller or programmed off-line a priori. The graphical representation of Eq. (10) is given in Fig. 8. Unfortunately, simply changing the droop gains leads to different and dynamically unbalanced droop controllers with different time constants as shown later on. Constant speed of the shaft on power sharing transients can be achieved if and only if the collective sharing regulator transfer function is kept constant:

$$
\sum_{j}^{n} G_{E Q j}(s)=n G_{E Q j}^{(E S)}(s)=G_{E Q}(s)
$$

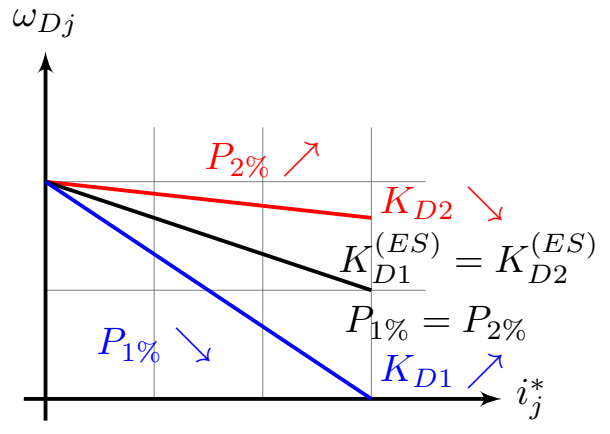

Fig. 8. Different coefficients produce different amount of torque

Eq. (11) and (7) empathise that whenever $K_{D j}^{(E S)}$ is updated, $K_{i S j}^{(E S)}$ must be updated accordingly. Provided that $\sum_{j}^{n} P_{j}=$ 1, Eq. (11) can be satisfied by dividing the individual equal power droop coefficients $K_{D j}^{(E S)}$ by a factor:

$$
\xi_{j}=n P_{j}
$$

and multiplying the individual integral gain $K_{i S j}^{(E S)}$ by the same factor $\xi_{j}$ like in the following combining Eq. (3) and (11):

$$
\frac{n K_{i S j}^{(E S)}}{s+K_{i S j}^{(E S)} K_{D j}^{(E S)}}=\sum_{j}^{n} \frac{\overbrace{K_{i S j}^{(E S)} \xi_{j}}^{K_{i S j}}}{s+\underbrace{K_{i S j}^{(E S)} \xi_{j}}_{K_{i S j}} \underbrace{\frac{K_{D j}^{(E S)}}{\xi_{j}}}_{K_{D j}}}
$$

Fig. 8 shows a graphical representation of the power sharing change for the case $n=2$, referring to the single-module droop characteristics. As a final result, changing the power contribution of module 1 from $0.5[p . u$.$] (equal sharing) to an$ arbitrary $P_{1}$ can be achieved dividing the droop slope $K_{D_{1}}$ by $\xi_{1}=n P_{1}$ and dividing the slope $K_{D_{2}}$ by $\xi_{2}=n\left(1-P_{1}\right)=$ $n P_{2}$. Since the current sharing dynamic is governed by the droop controller $G_{E Q j}$, its closed loop time constant in Eq.(14) dominates the power sharing transient.

$$
\tau_{\text {sharing }, j}=\frac{1}{K_{D j} K_{i S j}}
$$

For this reason, in order to keep constant the sharing regulator transfer function, both the integral gains $K_{i S j}^{(E S)}$ must be scaled accordingly like in Eq. (13). In Fig. 9, the two $i_{q}$ currents after a sharing ratio step (from equal sharing to $0.75 \% \div 0.25 \%$ ) are reported. It is possible to notice how without updating the integral gains $\left(K_{i S j}\right)$ of the sharing regulators, different time constant ( $\tau_{\text {sharing }, j}$ from (14)) are obtained. This leads to total flowing current variation within the electrical motor during the transient. In fact, $i_{q T O T}=i_{q 1}+i_{q 2}$ is not constant along the power sharing variation. This is reflected on the angular speed of the shaft in Fig. 10. Updating the integral gains, the speed is tracking the set-point during power sharing transients. Moreover, Eq. (14) confirms that updating the integral gain maintains constant sharing dynamic and guarantees constant 


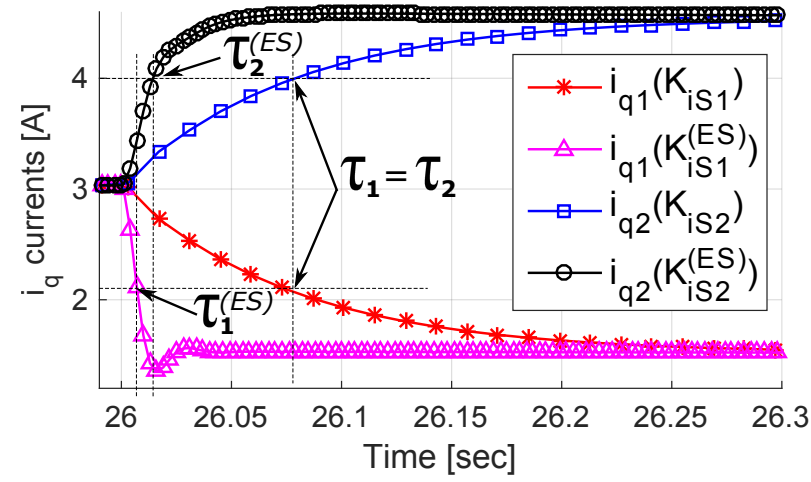

Fig. 9. Current sharing dynamic with and without updating the ingral gains $K_{i S j}$. Sharing ratio from $0.5 \% \div 0.5 \%$ (equal sharing) to $0.75 \% \div 0.25 \%$.

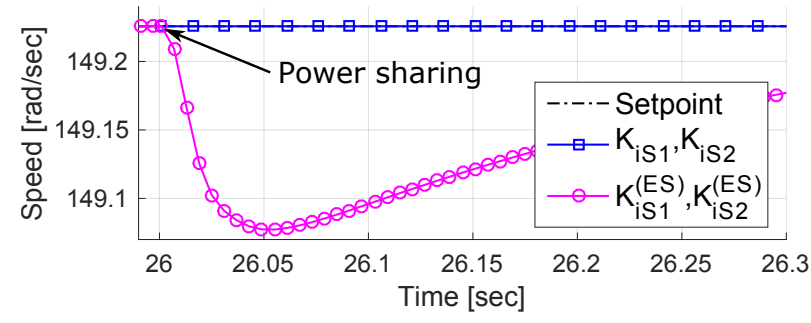

Fig. 10. Updating the integral gains, the final speed is not affected.

stability margins. This will be confirmed by experimental results in Sec. VIII.

\section{CONTROL DESIGN APPROACH}

Based on the previous discussion, and maintaining the same approximations, a simple design procedure can be proposed for the IMMD control. The inner current loop is designed to guarantee maximum bandwidth $\left(\omega_{c}\right)$. Then, the collective droop coefficient $K_{D}$ must be chosen with Eq. (15) (see Fig. 4a) where $\Delta \omega_{M A X}=\left(\omega^{*}-\omega\right)$ is the steady state speed error (without compensation PI) at full load.

$$
K_{D}=\Delta \omega_{M A X} /\left(\sum_{j}^{n} I_{n o m_{j}}\right)
$$

Referring to Fig. 7, the collective integral gain $K_{i S}$ can be calculated imposing the phase margin $\left(\varphi_{m}\right)$ with the following:

$$
\angle G_{E Q}(s) G_{I}(s) K_{t} G_{M}(s)=\varphi_{m}-\pi
$$

where $G_{M}(s)=1 /(s J+F)$ is the transfer function of the mechanical load, $K_{t}$ is the machine constant $\left(K_{t}=T / i\right)$, $G_{I}(s)=\omega_{c} /\left(s+\omega_{c}\right)$ is the closed loop current control. The design of the compensation PI can be achieved with aid of the equivalent collective scheme in Fig. 7. The plant for designing $P I_{D}$ is the total inner drooped loop transfer function $H_{E Q}(s)$, that can be written as:

$$
\begin{gathered}
H_{E Q}(s)=T_{E Q}(s) /\left(1+T_{E Q}(s)\right) \\
T_{E Q}(s)=G_{E Q}(s) G_{I}(s) K_{t} G_{M}(s)
\end{gathered}
$$

Under the previous hypothesis of balanced load and provided that $G_{E Q}=n G_{E Q j}^{E S}$, the same response of the designed equivalent collective system when using $n$ modules can be achieved multiplying by $n$ the collective droop gain and dividing by $n$ the collective integral gain (Eq. (8)).

Referring to Sec. $\mathrm{V}$, whenever the power sharing ratio has to be changed, power can be shared updating accordingly the sharing regulator parameters by the scaling factor $\xi_{j}$ (Eq. (12)).

\section{CONTROL DESIGN - CASE STUDY}

The system has been designed considering a set-point speed $\omega^{*}=149.2[\mathrm{rad} / \mathrm{sec}]$ equal to the nominal speed of the motor used for the experimental validation in Sec. VIII. Referring to the equivalent collective scheme in Fig. 7., the current controller has been assigned a bandwidth $B W_{C}=300[\mathrm{rad} / \mathrm{sec}]$ and a phase margin $P M_{C}=60^{\circ}$, whereas the outer loop $\left(P I_{D}\right)$ has been tuned with a bandwidth $B W_{D}=30[\mathrm{rad} / \mathrm{sec}]$ and a $P M_{D}=60^{\circ}$. The droop loop, or sharing loop, has been set up with different bandwidth $\left(B W_{D}<B W_{S}<B W_{C}\right)$ and phase margin $\left(P M_{S}\right)$ values in order to highlight and validate how the proposed strategy is able to control the current sharing dynamic. The collective droop gain coefficient can be obtained with Eq. (15) imposing $\Delta \omega_{M A X}$. This would be the steady state output speed of the system without the compensation $P I_{D}$. In this particular case, the maximum delta has been set up equal to the $15 \%$ of the reference speed $\left(\Delta \omega_{M A X}=22.38[\mathrm{rad} / \mathrm{sec}]\right)$. Considering a nominal current of $6.13[A]$, Eq. (15) leads to the collective droop coefficient $K_{D}=3.65[(\mathrm{rad} / \mathrm{sec}) / A]$. In the first results, the sharing loop has been set with $B W_{S}=40[\mathrm{rad} / \mathrm{sec}]$ and $P M_{S}=60^{\circ}$. The impact of this choice on the sharing dynamic will be discussed later in Sec. VIII-C.

The parameters required for the design have been estimated from the experimental setup with two motors on the same shaft presented in Sec. VIII. The magnetizing inductance and the stator resistance of the two motor sections have been measured with the no-load and short-circuit tests. The inertia and the friction of the system have been obtained from the data-sheets and measuring the time constant of the shaft. All the parameters are reported in Table II.

TABLE II

ESTIMATED MACHINE PARAMETERS

\begin{tabular}{|c|c|}
\hline Stator Inductance $L[H] \&$ Resistance $R[\Omega]$ & $0.257,3.7$ \\
\hline Shaft inertia $J\left[N m s^{2}\right] \&$ Friction $F[N m s]$ & $3 e^{-1}, 9 e^{-2}$ \\
\hline Machine constant $K_{t}[N m / A]$ & 3.27 \\
\hline
\end{tabular}

\section{EXPERIMENTAL VALIDATION}

The proposed model has been validated with the experimental rig in Fig. 11. The IMMD system has been emulated using three independent induction machines on the same shaft. In this configuration the mutual interaction between the threephase sets of windings are neglected. The first two machines are controlled as an equivalent IMMD with $n=2$ fed by a custom two level inverter [8], switching at $f_{S W}=5 \mathrm{kHz}$ and with DC bus at $V_{D C}=540 \mathrm{~V}$. The third motor is controlled as a load with a commercial inverter (Unidrive SP by Emerson). 


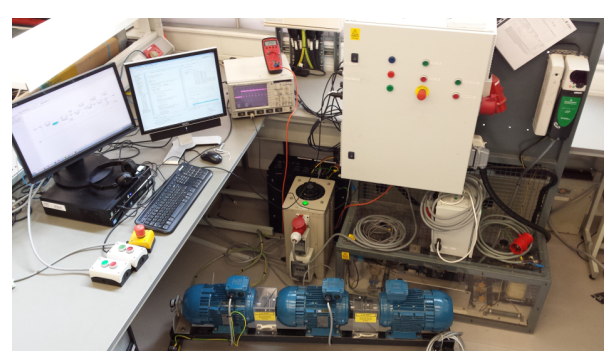

Fig. 11. Experimental rig

The three motors are identical and their plate data is reported in Table III.

TABLE III

MOTORS PLATE DATA

\begin{tabular}{|c|c|c|c|c|c|}
\hline $\mathrm{V}$ & $\mathrm{Hz}$ & $\mathrm{kW}$ & $\mathrm{rpm}$ & $\mathrm{A}$ & $\cos (\phi)$ \\
\hline 380 & 50 & 3.0 & 1425 & 6.13 & 0.85 \\
\hline
\end{tabular}

The custom inverter is controlled by a DSP/FPGA platform. Even if the controller is unique, the control loops for the two modules have been written independently without sharing information, to emulate the condition where the modules are completely independent.

\section{A. Collective equivalent module and speed dynamic}

The first experimental validation has been the implementation of the equivalent collective system in Fig. 7, and designed in Section VII, without the compensation loop. The obtained current and sharing controller parameters have been scaled by $n$ according to the discussion in Section VII, hence the droop coefficients become $K_{D_{1}}^{(E S)}=K_{D_{2}}^{(E S)}=n K_{D}=$ $2 * 3.65=7.3[(\mathrm{rad} / \mathrm{sec}) / A]$, whereas $K_{i S 1}^{(E S)}=K_{i S 2}^{(E S)}=$ $K_{i S} / n=26 / 2=13$. Fig. 12 reports experimental and

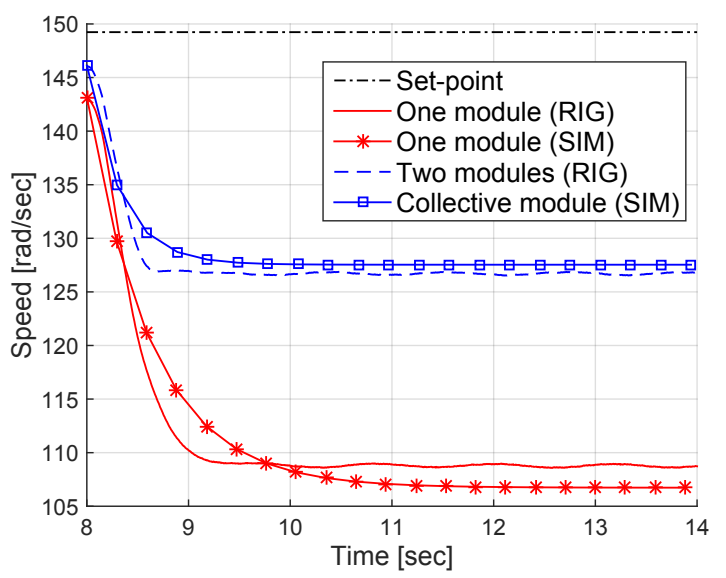

Fig. 12. Experimental validation of the design control loops for the equivalent collective not compensated system from zero to full load step $T_{L}=17 \mathrm{Nm}$

simulated response of the drive speed from a zero to a full load step $T_{L}=T_{L_{N O M}}=17[\mathrm{Nm}]$ to verify the response of the real system in the same conditions of the designed and simulated one. The speed reference is $\omega^{*}=149.2[\mathrm{rad} / \mathrm{sec}]$ and the load torque $T_{L}$ has been attached after 8 seconds. Looking at the figure it is possible to appreciate how the collective simulated module (blue line with squared markers) presents similar dynamics of the two motors in the experiment (dashed blue line). Representing the case of $n=2$, if one of the module fails, the continuous red line and the one with the asterisk markers are respectively the real and simulated speed of one single motor with scaled values. The steady state values, with and without $T_{L}$, can be verified with Eq. (4) and Eq. (15) either. In Fig. 13 the speeds under the same conditions of Fig. 12, except for the addition of the compensation loop, are reported.

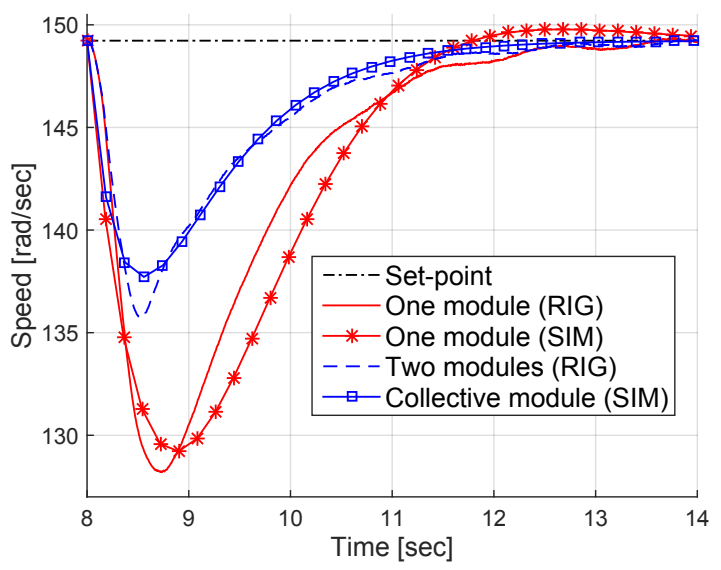

Fig. 13. Experimental validation of the design control loops for the equivalent collective compensated system from zero to full load step $T_{L}=17 \mathrm{Nm}$

\section{B. Current sharing dynamic}

The second step of the experimental validation is the implementation and prediction of the current sharing dynamic in response to a change in an external sharing command. In order to do so, the emulated IMMD is brought to steady state with equally shared power (3.06 $[A]$ per motor) and full load torque $T_{L_{N O M}}=17[\mathrm{Nm}]$. At $t=8[\mathrm{~s}]$ the power sharing is changed to $25 \%$ to module 1 and $75 \%$ to module 2 , i.e. $K_{D_{1}}=K_{D_{1}}^{(E S)} / \xi_{1}=7.3 /(2 * 0.25)=14.6$ and $K_{D_{2}}=$ $K_{D_{2}}^{(E S)} / \xi_{2}=7.3 /(2 * 0.75)=4.86$. Moreover, for keeping constant the droop loop gain, the integral gains have been scaled accordingly $\left(K_{i S 1}=K_{i S 1}^{(E S)} \xi_{1}=13 * 2 * 0.75=19.5\right.$ and $\left.K_{i S 2}=K_{i S 2}^{(E S)} \xi_{2}=13 * 2 * 0.25=6.5\right)$. Fig. 14 shows the current sharing dynamic. Based on the discussion in Section $\mathrm{V}$, these sharing dynamics can be predicted thanks to (14). Looking at the steps at $t=8[\mathrm{sec}]$, the $63 \%$ of the relative steps from $i_{q}=3.06 \mathrm{~A}$ to the final values $(4.59 \mathrm{~A}-1.53 \mathrm{~A})$ are relatively, in terms of absolute current, $4.02 A$ and $2.09 A$. Table IV reports all the values of $K_{i S j}, K_{D j}$, and the relative time constants computed with (14). The calculations are validated for every experiment and simulation in Fig.14. It is worth to mention the values of $\tau_{1}^{(E S)}$ and $\tau_{2}^{(E S)}$ are different because 


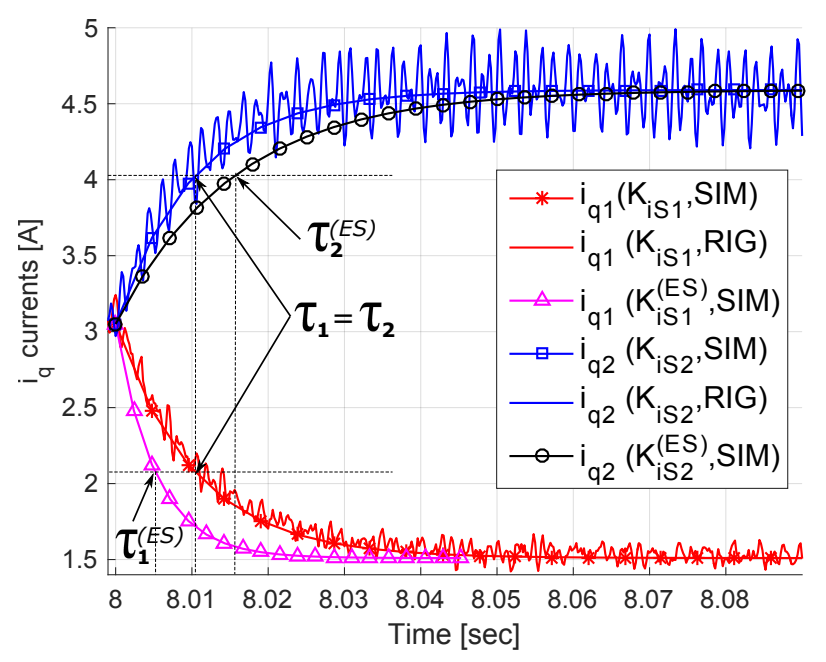

Fig. 14. Current sharing with $\left(\tau_{1}, \tau_{2}\right)$ and without $\left(\tau_{1}^{(E S)}, \tau_{2}^{(E S)}\right)$ updating the integral gain of the droop loop. Before second $8^{t h}$, the power is equally split (3.06A per motor). At $t=8 \mathrm{sec}$ the power is split with a $75 \%-25 \%$ ratio $(4.59[\mathrm{~A}]-1.53[\mathrm{~A}])$

TABLE IV

SHARING CONTROLLER PARAMETERS AND TIME CONSTANTS

\begin{tabular}{|c|c|c|c|c|}
\hline$P_{\% j}$ & $K_{i S j}$ & $K_{D j}$ & $\tau_{x}[\mathrm{sec}]-8 \mathrm{sec}$ & $\mid 63 \%$ relative step $\mid[A]$ \\
\hline $50 \%$ & 13 & 7.3 & & 3.06 \\
\hline $25 \%$ & 6.5 & 14.6 & $0.01=\tau_{1}$ & 2.09 \\
\hline $75 \%$ & 19.5 & 4.86 & $0.01=\tau_{2}$ & 4.02 \\
\hline $25 \%$ & 13 & 14.6 & $0.005=\tau_{1}^{(E S)}$ & 2.09 \\
\hline $75 \%$ & 13 & 4.86 & $0.015=\tau_{2}^{(E S)}$ & 4.02 \\
\hline
\end{tabular}

the sharing loop gain has not been kept constant. This means that the sum of the currents $\left(i_{q T O T}=i_{q 1}+i_{q 2}\right)$ are not constant during the transient affecting the final speed of the shaft as explained in the next subsection.

\section{Shaft speed sensitivity to power sharing}

It must be noticed however, by not updating the integral gain of the droop loop, the sharing time constants (Eq.(14)) are different for the two modules. This difference is reflected in the angular speed of the shaft as highlighted in Fig. 15. The fully loaded system $T_{L}=17[\mathrm{Nm}]$ is brought to steady state with equally shared power, and at $t=8[\mathrm{sec}]$ the sharing ratio has been set to $25 \%-75 \%$, exactly like in Fig. 14. In this set of experiments, at $t=9[\mathrm{sec}]$ the ratio has been swapped $(75 \%-25 \%)$. Fig. 16 shows the same experiment with a different sharing loop bandwidth (in Fig. $16 B W_{S}=120[\mathrm{rad} / \mathrm{sec}]$, whereas in Fig. 15 $\left.B W_{S}=40[\mathrm{rad} / \mathrm{sec}]\right)$. Both the experiments have been run updating (red line with diamond markers) and not updating (continuous blue line) the integral gains of the two modules. As long as the $B W_{S}$ increases, the current sharing dynamics become faster. For $B W_{S}=B W_{C}$, the sharing dynamic would be the same obtained with configuration depicted in Fig. 6, where the current set-point can be changed by the gain $W_{j}$. A similar behaviour has been achieved in the experimental results by setting $B W_{S}=120[\mathrm{rad} / \mathrm{sec}]$ (2.5 times slower than $\left.B W_{C}\right)$. In this case, updating the integral gains during sharing transients is not needed (in Fig. 16 the continuous blue line matches the red one with diamond markers). However, fast sharing transients could lead to vibrations caused by the quasi-step torque change. If the application requires a smoother transition, the approach proposed in this paper offers the opportunity to control the sharing transient updating the integral gains still tracking the speed reference.

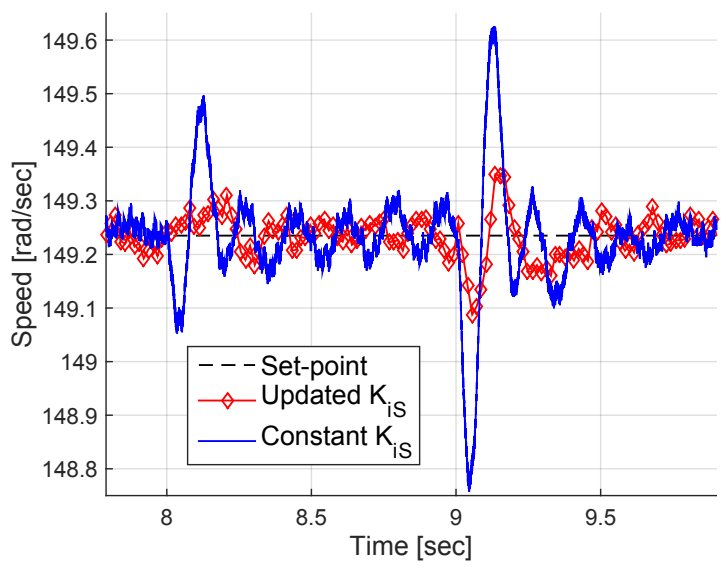

Fig. 15. The angular speed of the shaft with and without updating the integral gains $K_{i S j}$ with the slower sharing set-up $\left(B W_{S}=40 \mathrm{rad} / \mathrm{sec}\right)$

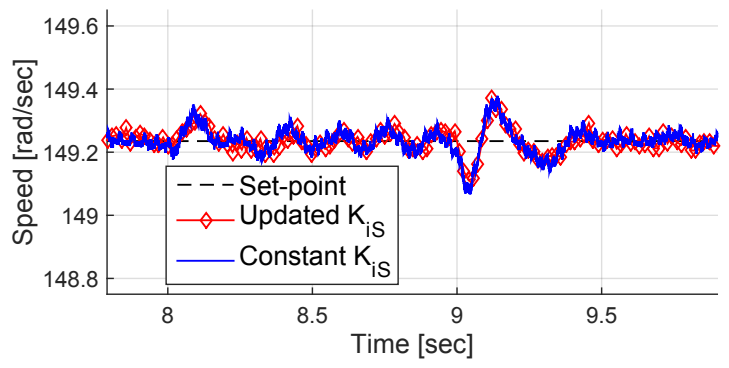

Fig. 16. The angular speed of the shaft with and without updating the integral gains $K_{i S j}$ with the faster sharing set-up $\left(B W_{S}=120 \mathrm{rad} / \mathrm{sec}\right)$

\section{Conclusions}

This manuscript is focused on Integrated Modular Motor Drives (IMMD) for high-reliability transportation applications. The suggested speed drooped system compared to the state of the art is more reliable being fully modular. The state space model of the system is realized and successfully verified. A simplified design procedure has been proposed for the tuning of the different control loops. The global speed dynamics has been designed first, and then attention has been focused on the power sharing transients. The whole procedure has been validated step by step in Matlab simulation first, and then with a $3 k W$ experimental rig showing good agreement with the expected results. The proposed system appears to be a good candidate for some applications of integrated modular motor drive for its inherent higher reliability and fault tolerance. 


\section{REFERENCES}

[1] Y. Shakweh, G. H. Owen, D. J. Hall, and H. Miller. Plug and play integrated motor drives. In Power Electronics, Machines and Drives, 2002. International Conference on (Conf. Publ. No. 487), pages 655661, June 2002.

[2] Jiyao Wang, Ye Li, and Yehui Han. Evaluation and design for an integrated modular motor drive (immd) with gan devices. In Energy Conversion Congress and Exposition (ECCE), 2013 IEEE, pages 43184325, Sept 2013.

[3] Jiyao Wang, Ye Li, and Yehui Han. Integrated modular motor drive design with gan power fets. Industry Applications, IEEE Transactions on, 51(4):3198-3207, July 2015.

[4] A.R. Iyer, R.P. Kandula, R. Moghe, J.E. Hernandez, F.C. Lambert, and D. Divan. Validation of the plug-and-play ac/ac power electronics building block (ac-pebb) for medium-voltage grid control applications. Industry Applications, IEEE Transactions on, 50(5):3549-3557, Sept 2014.

[5] M. Pulvirenti, G. Scarcella, G. Scelba, M. Cacciato, and A. Testa. Faulttolerant ac multidrive system. Emerging and Selected Topics in Power Electronics, IEEE Journal of, 2(2):224-235, June 2014.

[6] M.A Shamsi-Nejad, B. Nahid-Mobarakeh, S. Pierfederici, and F. Meibody-Tabar. Fault tolerant and minimum loss control of doublestar synchronous machines under open phase conditions. Industrial Electronics, IEEE Transactions on, 55(5):1956-1965, May 2008.

[7] S. Khwan-on, L. De Lillo, L. Empringham, P. Wheeler, C. Gerada, N.M. Othman, O. Jasim, and J. Clare. Fault tolerant power converter topologies for pmsm drives in aerospace applications. In Power Electronics and Applications, 2009. EPE '09. 13th European Conference on, pages 1-9, Sept 2009.

[8] L. de Lillo, L. Empringham, P.W. Wheeler, S. Khwan-on, C. Gerada, M.N. Othman, and Xiaoyan Huang. Multiphase power converter drive for fault-tolerant machine development in aerospace applications. Industrial Electronics, IEEE Transactions on, 57(2):575-583, Feb 2010.

[9] F. Farina, D. Rossi, A. Tenconi, F. Profumo, and S.E. Bauer. Thermal design of integrated motor drives for traction applications. In Power Electronics and Applications, 2005 European Conference on, pages 10 pp.-P.10, 2005.

[10] A. Tenconi, F. Profumo, S.E. Bauer, and M.D. Hennen. Temperatures evaluation in an integrated motor drive for traction applications. Industrial Electronics, IEEE Transactions on, 55(10):3619-3626, Oct 2008.

[11] Y. Tadros, J. Ranneberg, and U. Schafer. Ring shaped motor-integrated electric drive for hybrid electric vehicles. In European Power Electronics conference (EPE), Toulouse, France, September 2003.

[12] C. Klumpner and F. Blaabjerg. Short term braking capability during power interruptions for integrated matrix converter-motor drives. Power Electronics, IEEE Transactions on, 19(2):303-311, March 2004.

[13] Pw. Wheeler, J.C. Clare, M. Apap, L. Empringham, K.J. Bradley, S. Pickering, and D. Lampard. A fully integrated $30 \mathrm{kw}$ motor drive using matrix converter technology. In Power Electronics and Applications, 2005 European Conference on, pages 9 pp.-P.9, 2005.

[14] N. R. Brown, T.M. Jahns, and R.D. Lorenz. Power converter design for an integrated modular motor drive. In Industry Applications Conference, 2007. 42nd IAS Annual Meeting. Conference Record of the 2007 IEEE, pages 1322-1328, Sept 2007.

[15] A. Shea and T.M. Jahns. Hardware integration for an integrated modular motor drive including distributed control. In Energy Conversion Congress and Exposition (ECCE), 2014 IEEE, pages 4881-4887, Sept 2014.

[16] R. Bojoi, A. Cavagnino, A. Tenconi, and S. Vaschetto. Control of shaftline-embedded multiphase starter/generator for aero-engine. Industrial Electronics, IEEE Transactions on, PP(99):1-1, 2015.

[17] G. Scelba, G. Scarcella, M. Pulvirenti, M. Cacciato, A. Testa S. De Caro, and T. Scimone. Current-sharing strategies for faulttolerant ac multidrives. Industry Applications, IEEE Transactions on, 51(5):3943-3953, Sept 2015.

[18] A. Galassini, A. Costabeber, C. Gerada, G. Buticchi, and D. Barater State space model of a modular speed-drooped system for high reliability integrated modular motor drives. In Electrical Systems for Aircraft, Railway, Ship Propulsion and Road Vehicles (ESARS), 2015 International Conference on, pages 1-6, March 2015.

[19] A. Galassini, A. Costabeber, and C. Gerada. Speed droop control of integrated modular motor drives. In Industrial Electronics Society,
IECON 2015 - 41st Annual Conference of the IEEE, pages 003271003276 , Nov 2015.

[20] Allen-Bradley. Load sharing applications for the 1336 impact ac drive. 1336E-WP001A-EN-P, June 2000

[21] B. Jeftenic, M. Bebic, and S. Statkic. Controlled multi-motor drives. In Power Electronics, Electrical Drives, Automation and Motion, 2006. SPEEDAM 2006. International Symposium on, pages 1392-1398, May 2006.

[22] M.O.E. Aboelhassan, T. Raminosoa, A. Goodman, L. De Lillo, and C. Gerada. Performance evaluation of a vector-control fault-tolerant flux-switching motor drive. Industrial Electronics, IEEE Transactions on, 60(8):2997-3006, Aug 2013.

[23] T. Raminosoa, C. Gerada, and M. Galea. Design considerations for a fault-tolerant flux-switching permanent-magnet machine. Industrial Electronics, IEEE Transactions on, 58(7):2818-2825, July 2011.

[24] B.A Welchko, T.A Lipo, T.M. Jahns, and S.E. Schulz. Fault tolerant three-phase ac motor drive topologies: a comparison of features, cost, and limitations. Power Electronics, IEEE Transactions on, 19(4):11081116, July 2004.

[25] Wood Allen J., Wollenberg Bruce F., and Sheble Gerald B. Power Generation, Operation, and Control. Wiley, 1984.

[26] Qing-Chang Zhong. Robust droop controller for accurate proportional load sharing among inverters operated in parallel. Industrial Electronics, IEEE Transactions on, 60(4):1281-1290, April 2013.

[27] T.B. Lazzarin, G.A.T. Bauer, and I. Barbi. A control strategy for parallel operation of single-phase voltage source inverters: Analysis, design and experimental results. Industrial Electronics, IEEE Transactions on, 60(6):2194-2204, June 2013.

[28] B.K. Johnson, R.H. Lasseter, F.L. Alvarado, and R. Adapa. Expandable multiterminal dc systems based on voltage droop. Power Delivery, IEEE Transactions on, 8(4):1926-1932, Oct 1993.

[29] U. Borup, F. Blaabjerg, and P.N. Enjeti. Sharing of nonlinear load in parallel-connected three-phase converters. Industry Applications, IEEE Transactions on, 37(6):1817-1823, Nov 2001.

[30] S. D'Arco, A Petterteig, R. Pittini, and T.M. Undeland. Droop regulated vscs for island operation of future offshore systems. In PowerTech, 2011 IEEE Trondheim, pages 1-6, June 2011.

[31] D. Fingas and P.W. Lehn. Operation of parallel three-phase converters as a motor drive. In Industrial Electronics, 2009. IECON '09. 35th Annual Conference of IEEE, pages 1217-1222, Nov 2009. 